\title{
Article
}

\section{A clinical case series investigating the effectiveness of an exercise intervention in chronic inflammatory demyelinating polyneuropathy (CIDP)}

Janssen, Jessica, Bunce, Megan, Nixon, John, Dunbar, Amanda Jane, Jones, Sam, Benstead, Jackie, Jeanes, Yvonne, Selfe, James and Richards, James

Available at http://clok.uclan.ac.uk/21067/

Janssen, Jessica ORCID: 0000-0002-5961-2736, Bunce, Megan, Nixon, John, Dunbar, Amanda Jane, Jones, Sam, Benstead, Jackie, Jeanes, Yvonne, Selfe, James and Richards, James ORCID: 0000-0002-4004-3115 (2018) A clinical case series investigating the effectiveness of an exercise intervention in chronic inflammatory demyelinating polyneuropathy (CIDP). Physiotherapy Practice and Research, 39 (1). pp. 37-44. ISSN 2213-0683

It is advisable to refer to the publisher's version if you intend to cite from the work. http://dx.doi.org/10.3233/ppr-170100

For more information about UCLan's research in this area go to http://www.uclan.ac.uk/researchgroups/ and search for <name of research Group>.

For information about Research generally at UCLan please go to http://www.uclan.ac.uk/research/

All outputs in CLoK are protected by Intellectual Property Rights law, including Copyright law. Copyright, IPR and Moral Rights for the works on this site are retained by the individual authors and/or other copyright owners. Terms and conditions for use of this material are defined in the policies page. 
1 A clinical case series investigating the effectiveness of an exercise

2 intervention in chronic inflammatory demyelinating polyneuropathy (CIDP)

3 Jessie Janssen $\mathrm{PhD}^{\mathrm{a}}$, Megan Bunce ${ }^{\mathrm{a}}$, John Nixon $\mathrm{MD}^{\mathrm{b}}$, Mandy Dunbara, Sam

4 Jones $^{b}$, Jackie Benstead ${ }^{b}$, Yvonne Jeanes ${ }^{b}$, James Selfe DSc ${ }^{c}$, Jim Richards PhDa

$5 \quad$ a Allied Health Research unit, University of Central Lancashire, Preston, UK

6 b Royal Preston Hospital, Lancashire Teaching Hospitals NHS Foundation Trust,

7 Preston, UK

$8{ }^{c}$ Department of Health Professions, Manchester Metropolitan University,

9 Manchester, UK

11 Corresponding author: Dr Jessie Janssen, BB204, Research Fellow (Physiotherapy),

12 Allied Health Research unit, University of Central Lancashire, UK, Preston.

13 +441772894560, jianssen@uclan.ac.uk

14 Word count: 2712

15

Keywords

- Chronic inflammatory demyelinating polyneuropathy

- Exercise

- Physiotherapy

- Balance

- Gait 


\section{Abstract:}

2 Background: Despite clinical intervention, people with chronic inflammatory

3 demyelinating polyneuropathy (CIDP) experience difficulties in gait and balance on a

4 daily basis. However, the effects on these variables of a tailored home based

5 exercise programme for this population have not been investigated. This case series

6 aims to investigate the effects of a home based tailored exercise programme on gait

7 and balance in people with CIDP.

8 Methods: Case series of seven people with CIDP from a neurology department of a

9 local hospital. Participants took part in a 6 week Otago exercise programme, which

10 include walking, strengthening and balance tasks. Participants were assessed 10

11 times; 3 times prior, 3 times during an exercise intervention, 3 times post intervention

12 and once at three months follow up. The outcome measures were Berg Balance

13 scale, 10 meter walk test, fatigue severity scale and EQ-5D-5L.

14 Results: Participants showed an increase in walking speed and balance after the exercise intervention and most kept these improvements at 3 months follow up.

16 Conclusion: This study shows that exercise can be beneficial for gait and balance in people with CIDP. These findings are in line with literature from related diseases such as Guillain-Barre Syndrome. However studies with a larger sample size are needed to confirm these findings in the population. 
2 Chronic Inflammatory Demyelinating Polyneuropathy (CIDP) is a rare and

3 progressive autoimmune disease of the peripheral nervous system. Yearly up to

4 approximately 1.6 per 100,000 of the UK population contracts the disease, whereas

$58.9 / 100,000$ people are living with CIDP [1].

6 Characterized by distal and proximal weakness of the upper and lower limbs, the

7 disease also presents with sensory impairments such as balance disturbance, numbness of the hands and feet and areflexia [2]. However the presentation of the symptoms for each individual varies greatly, therefore the population is very heterogeneous. In addition, motor defects associated with the disorder may lead to abnormalities in gait, and the long term nature of this condition can lead to impairment in both psychological and social functioning.

Pharmaceutical approaches have shown the condition responds to treatment with intravenous-immunoglobulin (IVIG) as the primary treatment preference $[3,4]$, corticosteroids [5], and plasma exchange (plasmapheresis) [6]. Although these approaches can reduce the severity of disability in CIDP, administration of these interventions exposes significant clinical disadvantages such as being expensive, time-consuming and the development of potentially serious side effects with long term usage [7]. In addition, maintaining movement and balance throughout the duration of a treatment cycle may be challenging as the patient's condition may deteriorate over time. Despite the use of the medical treatments described above, clinicians see a significant proportion of patients suffering with moderate to severe disabilities on the longer-term. 
1 The benefits of an exercise programme have been shown in Acute Inflammatory

2 Demyelinating Polyneuropathy or Guillain-Barre Syndrome (GBS). Additional exercise conducted by a mildly affected patient with GBS showed considerable improvements in ground reaction forces and lower limb kinematics, indicating a markedly improved functional gait pattern. This improvement was reinforced by positive subjective feedback [8]. Further research comparing the impact between low-intensity and high-intensity ambulatory rehabilitation programme found a clinical improvement which favoured the high-intensity programme in GBS patients [9]. Additionally, physical therapy interventions applied to case studies in GBS have also shown marked improvements in muscle performance and functional independence [10]. In addition, exercise has been shown to improve other polyneuropathies in clinical measures of balance and postural instability [11]. Although the mechanisms that apply to the disease progression between CIDP and GBS are different, scope for physical improvement in recovery with GBS could still suggest a similar output if these practices were applied to CIDP patients.

There has been little research over the years providing information on the functional impact of exercise in CIDP, this is partly due to the rarity of the condition. The majority of the research has focused on the beneficial effects of exercise within lifestyle domains, such as improvement in quality of life [12] and activity limitation [13]. Additionally research has focussed on broader measures of health including fatigue and mental states such as anxiety and depression in people with CIDP [14]. Whilst these are important factors in CIDP, as they can significantly disrupt an individual's activity within daily living, there is a lack of information on the effect of physiotherapy interventions and their potential benefit on the physical aspects of mobility and disability. There is small amount of limited evidence that suggests that 
1 focusing on increasing general fitness in this patient group is sufficient for

2 rehabilitation. However, a case series in patients with CIDP found no relationship between physical fitness and the patient's actual mobility using a 12 week training

4 programme with a singular focus on aerobic training [15]. This could suggest that

5 patient's with CIDP may need an exercise programme which is more tailored to

6 specific patient's needs. Exercises will most likely vary between patients due to the

7 heterogeneous presentation of symptoms, with more focus on concentric and eccentric exercises with an intention to target problematic or weakened muscle groups to regain strength and balance.

Due to the nature in which the disorder presents itself, the variability of symptoms in this patient group is quite broad, making it considerably difficult to categorise CIDP patients as a group. A recent case report investigated a rehabilitation programme which targeted therapeutic exercise, balance training, and function training with progressive endurance activities in CIDP [16]. The Functional Independence

Measure was used as a clinical outcome measure; commonly used to measure disablement and level of independence in patients. The patient with CIDP had an and balance patients with CIDP.

\section{Methods}

Ethics 
1 This study was approved by the University Ethics Committee \{number\} and the

2 National Research Ethics Service committee \{number\}

3 Participant recruitment

4 A total of 7 participants over the age of 18 with a diagnosis of CIDP or other acquired

5 inflammatory polyneuropathy and who were on a stable IVIG cycle, between 1-8

6 weeks consistently for 3 months or more prior to the study, were recruited into the

7 study. Participants previously received treatment as patients at the local hospital, and had been diagnosed by a consultant neurologist using clinical assessment and

9 standard neurophysiological tests.

Exclusion criteria included; no detectable leg weakness, inadequate vision or hearing to receive instructions, diagnosis of multifocal motor neuropathy, any significant relevant comorbidity e.g. significant arthritis, cardiac failure, cervical myelopathy which might affect gait, the need to use ambulatory aids during walking and any inconsistent IVIG use throughout the study.

Each participant was presented with information about the study and their right to withdraw, and gave their full consent to participate by completing a signed consent form. An Overall Neuropathies Limitation Scale (ONLS) assessment was carried out prior to the first assessment. The ONLS score was calculated by adding the arm score to the leg score and ranged from 0 (no disability) to 12 (maximum disability).

Protocol

21 This clinical case series was based on an AAABBBAAAA design; participants were assessed three times prior to, three times during, three times post intervention and once at three months post intervention. The assessment schedule was matched to 
1 each individual's IVIG treatment cycle, on average this meant that the first post

2 intervention assessment was measured three weeks after the intervention was completed. At each of the 10 assessments four outcome measures were administered; Berg Balance scale, 10 meter walk test (10MWT), Fatigue Severity Scale (FSS), and the EQ5D-5L. Six assessments took place at a clinical rehabilitation unit and four were carried out in a movement laboratory where additional gait analysis took place. The four laboratory assessments were conducted during each of the assessment periods; pre-intervention, during the intervention, post-intervention and at the 3 month follow up. (Figure 1)

\section{Berg Balance scale}

The Berg Balance scale $[17,18]$ is a clinical instrument designed to measure balance performance. Used as an outcome measure, the Berg Balance scale compromises of a range of 14 balance tasks (e.g. standing on one leg, tandem stepping).

Participants were instructed to perform each of the balance tasks in a standardised order. Berg Balance results were recorded on a 5-point ordinal scale, ranging from 0 4 , with a maximum possible score of 56 . Lower scores were associated with more limited balance in participants. In each assessment the Berg Balance scale was carried out once.

\section{Meter walk test}

Participants undertook three 10 MWT during each of the 10 assessments.

Participants were instructed to walk the length of a 10 meter walk way at their own comfortable speed. Three consecutive 10 MWT were carried out per assessment, with the middle 6 meters marked out and timed. The average time score was then taken from the three walks. 
2 The FSS [19] and the EQ-5D-5L health questionnaire [20] were completed based on

3 how the participants felt that week. The FSS compromises of 9-item questionnaire

4 relating to fatigue severity on daily living activities. Each question is scored from a

5 range of 0 (strongly disagree) to 7 (strongly agree), with a maximum score of 63 as a

6 whole. Additionally participants rated their global fatigue on a visual analogue scale 7 (VAS).

9 The EQ-5D-5L presents a range of 5 sub sections which include Mobility, Self-care, 10 Usual activities, Pain/Discomfort and Anxiety/Depression. Patients were asked to tick 11 which box applied to them (e.g. I have no problems/slight problems/moderate problems/severe problems or unable) within each specific section. The patient's perceived general health (EQ-5D-5L) was rated from 0 (being the worst) to 100

14 (being the best they have felt).

Data analysis

Individual 10MWT scores were compared with Minimal Clinically Important

17 Differences (MCID) scores obtained from the literature. The MCID was set to

$0.05 \mathrm{~m} / \mathrm{s}$ for a small meaningful change and $0.13 \mathrm{~m} / \mathrm{s}$ for a substantial meaningful change [21]. Conradsson et al 2007 identified that a change of 7.7 points on the Berg Balance scale marked a genuine change in function for a participant [22] and this cut off point was used in this study.

For the complete patient group average scores were taken from the 3 assessments in the pre-intervention assessment phases and the 3 assessments in the post- 
1 intervention phases for each of the clinical outcome measures. A 10\% increase was

2 set as a meaningful difference between pre- and post-intervention. Paired t-tests

5 Intervention

6 The Otago Home Exercise programme [23] was used as the intervention in this

\section{Insert Figure 1 here}


1 Seven participants (5 females, 2 males), participated in this study. One participant

2 withdrew from the study during the pre-intervention phase for personal reasons.

3 Therefore results are presented of the six remaining participants. One of these six

4 participants was unable to complete the 3 month post-assessment follow up, due to

5 unrelated health issues. Participants were between the ages 48 and 77 years old,

6 and scored 3 to 5 on the ONLS score. None of the participants changed their IVIG

7 treatment during the study.

$8 \quad$ Individual results

9 Despite a varied response in walking speed and balance during the intervention,

10 participants presented an overall trend towards an increase in walking speed as a

11 result of the exercise programme (Table 1, Figure 2). One of the participants

12 (participant 1) commented that her walking distance improved so greatly that she

13 was able to walk outside for leisure again. The majority of scores still displayed

14 either a small or substantial meaningful change in speed at 3 month follow up, with

15 the exception of participant 7 who showed no change in walking speed across the 16 assessments. 
4 All participants displayed an increase in balance in scores post-exercise, with the exception of participant 2 who hit the ceiling of the Berg balance score (56) during

6 intervention and maintained this maximum score for the duration of the study (Figure

7 3). This latter participant also commented that she was able to pick up her favourite

8 sport and therefore improve her participation level. The majority of participants

9 continued to improve in their balance scores 3 months post intervention. Here it must be noted that all of the participants continued all or part of their exercises after the

11 intervention was completed.

\section{Group results}

13 As a patient group, both of the clinical outcomes for the 10 MWT and the Berg

14 Balance test presented a statistically significant improvement in scores, displaying a $10 \%$ improvement in outcome post intervention in comparison to pre-intervention. A $10 \%$ improvement in scores were also seen in the secondary outcome measures

17 FSS and the EQ-5D-5L, however these changes were not statistically significant 18 (Table 2).

\section{Discussion}


1 This study showed that a six week tailored exercise intervention can have a positive

2 effect on walking speed and balance in people with stable CIDP. This effect was apparent immediately after the intervention and 3 months after the exercises were completed. IVIG treatment did not change for any of the participants in this time period. These results resonate with findings of others who also showed improvement

6 in functional independence [10] and balance [11] in people with related diseases.

7 During the intervention a varied response was seen regarding walking speeds and 8 balance. This variation might be explained by the challenging nature of the balance and walking exercises, with which some participants initially struggled. This is the first time that the Otago Exercise programme has been used in this population. In light of the success of this study it can be concluded that this programme is suitable extent this programme could be effective.

Despite the varied response of the exercise intervention it appeared that the 
1 feedback. Therefore even though the use of this scale is accepted in CIDP, it did not capture the limitations of the participants as full marks did not mean that the participant did not experience any issues [27].

4

In line with Graham et al [14] our participants also reported to be less fatigued, although this was not significant. A study encompassing more participants and perhaps a longer or more intense intervention could show a significant result. The reduced fatigue levels in this study could be caused by increased strength levels due

9 to the intervention, but could also be explained by the possibility that participants were less depressed due to improved Quality of Life, became less depressed, became less anxious of the assessments over time or by the day to day variability seen in this disease [14]. Although the latter is possible this study used an average of three assessments and assessed the participants at set time points to limit this effect.

\section{Study limitations}

The MCID values used in this study were taken from the literature for older people $[21,22]$. Despite of some similarity between this group and the CIDP participants, there are great differences between them as well. Therefore the effects of the intervention observed in this study would be more realistic if specific MCID values for the 10MWT and the Berg Balance scale for people with polyneuropathies existed.

Although case studies are suitable methodological approaches for such a rare and variable disorder, a larger sample size would be needed to consider an overall generalization of an exercise program in this patient group. This may explain why we have seen the beneficial effects in tailored exercise programmes within individual 
1 case studies, but there is a lack of evidence to promote exercise as useful for

2 recovery in CIDP as a whole group.

3

\section{Conclusion}

5 Tailored exercises can have a positive effect on walking speed and balance in

6 people with CIPD. However, further research on a bigger study population is needed

7 to identify which aspects facilitate this change.

8

$9 \quad$ Acknowledgements

10 We would like to thank all the participants involved and Glasshouse Appeal

11 (registered charity 1106441) for funding this study. 
1 Ethical Approval: This study was approved by the University Ethics Committee

$2(\mathrm{BuSH})$ and the National Research Ethics Service committee North West- Lancaster

3 reference number: 13/NW/0175

4

5 Funding: Glasshouse Appeal (registered charity 1106441) funded this study.

6

7 The authors declare no conflicts of Interest.

8 
1. Laughlin RS, Dyck PJ, Melton LJ, Leibson C, Ransom J, Dyck PJB. Incidence and prevalence of CIDP and the association of diabetes mellitus. Neurology 2009 73(1), 39-45.

2. Van den Bergh PY, Hadden RD, Bouche P, Cornblath DR, Hahn A, Illa I, Koski CL, Leger JM, Nobile-Orazio E, Pollard J, Sommer C, van Doorn PA, van Schaik IN. European Federation of Neurological Societies/Peripheral Nerve Society guideline on management of chronic inflammatory demyelinating polyradiculoneuropathy: report of a joint task force of the European Federation of Neurological Societies [trunc]. Eur J Neurol. 2010 Mar;17(3):356-63.

3. Van Doom PA, Brand A, Strengers PF. High-dose intravenour mmunoglobulin treatment in Chronic Inflammatory Demyelianting Polyneuropath: a doubleblind placebo-controlled, crossover stud. Neurology 1990, 40: 209-212.

4. Hughes RA, Donofrio P, Bril V, Dalakas MC, Deng C, Hanna K, Hartung HP, Latov N, Merkies IS, van Doorn PA; ICE Study Group. Intravenous immune globulin ( $10 \%$ caprylate-chromatography purified) for the treatment of chronic inflammatory demyelinating polyradiculoneuropathy (ICE study): a randomised placebo-controlled trial. Lancet Neurol. 2008 Feb;7(2):136-44.

5. Dyck PJ, O'Brien PC, Oviatt KF, Dinapoli RP, Daube JR, Bartleson JD, Mokri B, Swift T, Low PA, Windebank AJ. Prednisone improves chronic inflammatory demyelinating polyradiculoneuropathy more than no treatment. Ann Neurol, 1982. 11:136-141,19

6. Hughes R, Bensa S, Willison H, Van Den Bergh P, Comi G, Illa I, NobileOrazio E, Van Doorn P, Dalakas M, Bojar M, Swan A, Randomized controlled 
trial of intravenous immunoglobulin versus oral prednisolone in chronic inflammatory demyelinating polyradiculoneuropathy. Ann Neurol. 2001 50: 195-201.

7. Dyck PJ. Intravenous Immunoglobulin in chronic inflammatory demyelinating polyradiculoneuropathy and in neuropathy associated with IgM Monoclonal gammopathy of unknown significance. Neurology 1990;40:327-8.

8. Walton T, Vincent M, Richards J, Davidson I. Usefulness of digital gait analysis for assessing patients with Guillain-Barre syndrome. International Journal of Therapy and Rehabilitation. 2005;12(9):388-393.

9. Khan F, Pallant JF, Amatya B, Ng L, Gorelik A, Brand C. Outcomes of highand low-intensity rehabilitation programme for persons in chronic phase after Guillain-Barré syndrome: a randomized controlled trial. J Rehabil Med. 2011 Jun;43(7):638-46.

10. Fisher TB, Stevens JE. Rehabilitation of a marathon runner with GuillainBarré syndrome. J Neurol Phys Ther. 2008 Dec;32(4):203-9.

11. Richardson JK, Sandman D., and Vela S. A focused exercise regimen improves clinical measures of balance in patients with peripheral neuropathy. Arch Phys Med Rehabil 2001 Feb;82(2):205-9.

12. Garssen MP, Bussmann JB, Schmitz PI, Zandenbergen A, Welter TG, Merkies IS. Physical training and fatigue, fitness and quality of life in GuillainBarre syndrome and CIDP. Neurology 2004: 63:2393-2395.

13. White CM, Hadden RD, Robert-Lewis SF, McCrone PR, Petty JL. Observer blind randomised controlled trial of a tailored home exercise programme versus usual care in people with stable inflammatory immune mediated neuropathy. BMC Neurol. 2015 Aug 21;15:147 
14. Graham RC, Hughes RA, White CM. A prospective study of physiotherapist prescribed community based exercise in inflammatory peripheral neuropathy. J Neurol. 2007 Feb; 254(2):228-35.

15. Bussmann JB, Garssen MP, van Doorn PA, Stam HJ. Analysing the favourable effects of physical exercise: relationships between physical fitness, fatigue and functioning in Guillain-Barré syndrome and chronic inflammatory demyelinating polyneuropathy. J Rehabil Med. 2007 Mar;39(2):121-5

16. Chong DY, Glickman LB, Cabanero-Johnson PS. Chronic Inflammatory Demyelinating Polyradiculoneuropathy from a Physical Therapist's perspective: A case report. Journal of Acute Care Physical Therapy 2010. 1(1), 4-13.

17. Berg K, Wood-Dauphinee S, Williams Jl, Gayton D. Measuring balance in the elderly: Preliminary development of an instrument. Physiotherapy Canada 1989 41:304-311.

18. Berg K, Wood-Dauphinee S, Williams JI, Maki, B. Measuring balance in the elderly: Validation of an instrument. Can. J. Pub. Health, 1992 July/August supplement 2:S7-11

19. Krupp LB, LaRocca NG, Muir-Nash J, Steinberg AD. The fatigue severity scale. Application to patients with multiple sclerosis and systemic lupus erythematosus. Arch Neurol. 1989 Oct;46(10):1121-3

20. EuroQol Group. EuroQol--a new facility for the measurement of health-related quality of life. Health Policy. 1990 Dec;16(3):199-208.

21. Perera S, Mody SH, Woodman RC, Studenski SA. Meaningful change and responsiveness in common physical performance measures in older adults. $\mathrm{J}$ Am Geriatr Soc. 2006 May;54(5):743-9. 
22. Conradsson $M$, Lundin-Olsson L, Lindelöf $\mathrm{N}$, Littbrand $\mathrm{H}$, Malmqvist $\mathrm{L}$, Gustafson Y, Rosendahl E. Berg balance scale: intrarater test-retest reliability among older people dependent in activities of daily living and living in residential care facilities. Phys Ther. 2007 Sep;87(9):1155-63.

23. Campbell AJ, Robertson MC, Gardner MM, Norton RN, Tilyard MW, Buchner DM. Randomised controlled trial of a general practice programme of home based exercise to prevent falls in elderly women. BMJ. 1997 Oct $25 ; 315(7115): 1065-9$.

24. Brusse KJ, Zimdars S, Zalewski KR, Steffen TM. Testing functional performance in people with Parkinson disease. Phys Ther. 2005 Feb;85(2):134-41.

25. Wirz M, Müller R, Bastiaenen C. Falls in persons with spinal cord injury: validity and reliability of the Berg Balance Scale. Neurorehabil Neural Repair. 2010 Jan;24(1):70-7.

26. Wang CH, Hsueh IP, Sheu CF, Yao G, Hsieh CL. Psychometric properties of 2 simplified 3-level balance scales used for patients with stroke. Phys Ther. 2004 May;84(5):430-8.

27. Blum L, Korner-Bitensky N. Usefulness of the Berg Balance Scale in stroke rehabilitation: a systematic review. Phys Ther. 2008 May;88(5):559-66. 
Table 1: average results of 10MWT, Berg Balance, FSS and EQ-5D-5L index for each participant

\begin{tabular}{|c|c|c|c|c|c|}
\hline \multicolumn{2}{|c|}{ Participant } & \multirow{2}{*}{$\begin{array}{l}\text { Pre } \\
1.18\end{array}$} & \multirow{2}{*}{$\begin{array}{l}\text { During } \\
\\
1.20\end{array}$} & \multirow{2}{*}{$\begin{array}{l}\text { Post } \\
\qquad 1.27^{\star}\end{array}$} & \multirow{2}{*}{$\begin{array}{r}3 \text { Month FU } \\
1.33^{\star \star}\end{array}$} \\
\hline 1 & 10MWT $(\mathrm{m} / \mathrm{s})$ & & & & \\
\hline & Berg Balance & 53.0 & 51.7 & 54.0 & 53.0 \\
\hline & FSS & 42.7 & 39.0 & 42.0 & 42.0 \\
\hline & EQ-5D (index) & 0.67 & 0.65 & 0.65 & 0.65 \\
\hline \multirow[t]{4}{*}{2} & 10MWT (m/s) & 1.13 & $1.26^{* *}$ & $1.31^{* *}$ & $1.26^{\star *}$ \\
\hline & Berg Balance & 47.0 & $55.7^{* * *}$ & $55.7^{* * \star}$ & $56.0^{* * *}$ \\
\hline & FSS & 32.7 & 17.7 & 12.3 & 11.0 \\
\hline & EQ-5D (index) & 0.80 & 0.84 & 0.89 & 0.84 \\
\hline \multirow[t]{4}{*}{4} & 10MWT (m/s) & 0.82 & 0.81 & 0.86 & $0.87^{*}$ \\
\hline & Berg Balance & 29.3 & 31.3 & 32.7 & 35.0 \\
\hline & FSS & 55.7 & 53.0 & 55.0 & 61.0 \\
\hline & EQ-5D (index) & 0.62 & 0.63 & 0.61 & 0.63 \\
\hline \multirow[t]{4}{*}{5} & 10MWT (m/s) & 0.46 & $0.55^{*}$ & $0.59^{* *}$ & $0.59^{\star \star}$ \\
\hline & Berg Balance & 21.0 & 21.0 & 22.5 & $29.0^{\star \star \star}$ \\
\hline & FSS & 50.7 & 49.3 & 50.5 & 50.0 \\
\hline & EQ-5D (index) & 0.47 & 0.45 & 0.45 & 0.38 \\
\hline \multirow[t]{4}{*}{6} & 10MWT (m/s) & 0.99 & 0.96 & $1.07^{*}$ & $\mathrm{n} / \mathrm{a}$ \\
\hline & Berg Balance & 49.0 & 50.0 & 53.7 & $\mathrm{n} / \mathrm{a}$ \\
\hline & FSS & 43.0 & 37.0 & 43.3 & $\mathrm{n} / \mathrm{a}$ \\
\hline & EQ-5D (index) & 0.63 & 0.66 & 0.57 & $\mathrm{n} / \mathrm{a}$ \\
\hline \multirow[t]{4}{*}{7} & 10MWT (m/s) & 0.80 & 0.77 & 0.84 & 0.78 \\
\hline & Berg Balance & 45.7 & 45.7 & 51.0 & 52.0 \\
\hline & FSS & 47.7 & 51.0 & 41.5 & 44.0 \\
\hline & EQ-5D (index) & 0.44 & 0.54 & 0.56 & 0.65 \\
\hline
\end{tabular}

$2 *$ indicates a small meaningful clinical change in walking speed compared to the pre-

3 condition, ** indicates a substantial meaningful clinical change in walking speed compared 4 to the pre-condition. $* * *$ indicates a genuine change in the Berg Balance test for a participant 5 compared to the pre-condition. No meaningful clinical differences were set a priori for the $6 \quad$ FSS and EQ-5D. 
1 Table 2: Differences and percentage change of 10MWT, Berg Balance, FSS and EQ-5D5L-

$2 \quad$ VAS for the 6 participants combined

\begin{tabular}{|l|l|l|}
\hline Outcome measure & p values & Percentage change \\
\hline $10 \mathrm{MWT}(\mathrm{m} / \mathrm{s})$ & $0.007^{*}$ & $10.4 \%$ \\
\hline Berg Balance & $0.016^{*}$ & $10.0 \%$ \\
\hline FSS & 0.220 & $-10.2 \%$ \\
\hline EQ-5D VAS & 0.323 & $12.2 \%$ \\
\hline
\end{tabular}

3 Results of paired t-test as a patient group for each clinical outcome measure pre vs post 4 intervention, ${ }^{*}$-value $<0.05$

5 


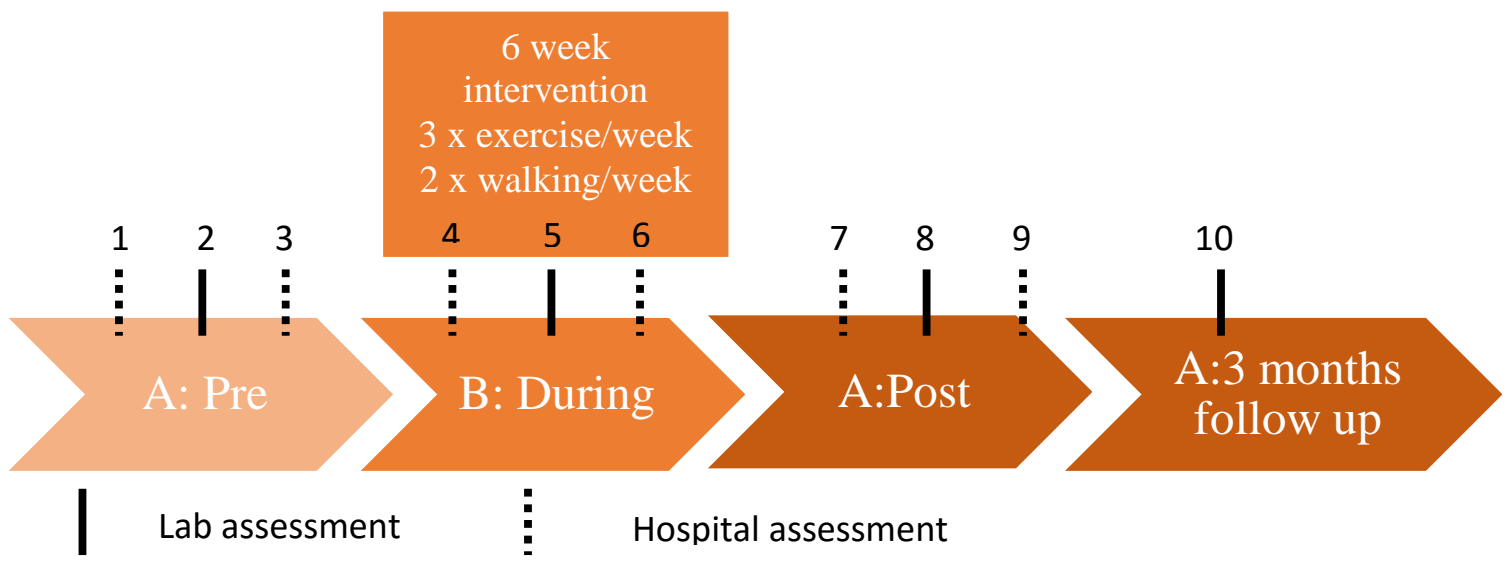

2

3 Figure 1: Overview of the 10 participant assessments and exercise intervention throughout 4 the study 


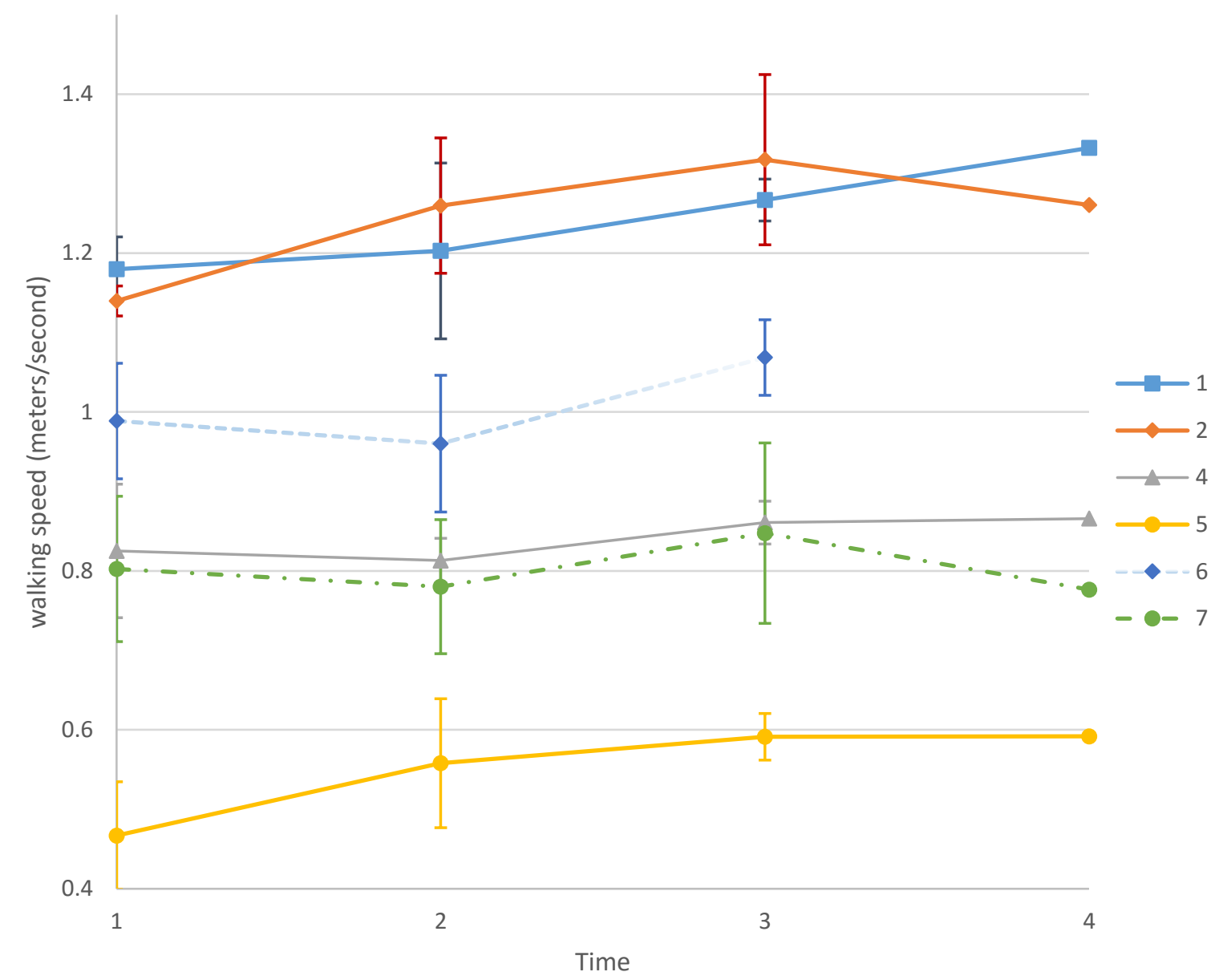

1

2 Figure 2: 10 meter walk values with standard deviation bars for each participant. The pre 3 (1), during (2), and post (3) values each represent the average of three assessments. The 3 4 month value (4) represents one assessment. Participant 6 was not able to complete the follow 5 up assessment at 3 months. 


\section{Berg Balance}

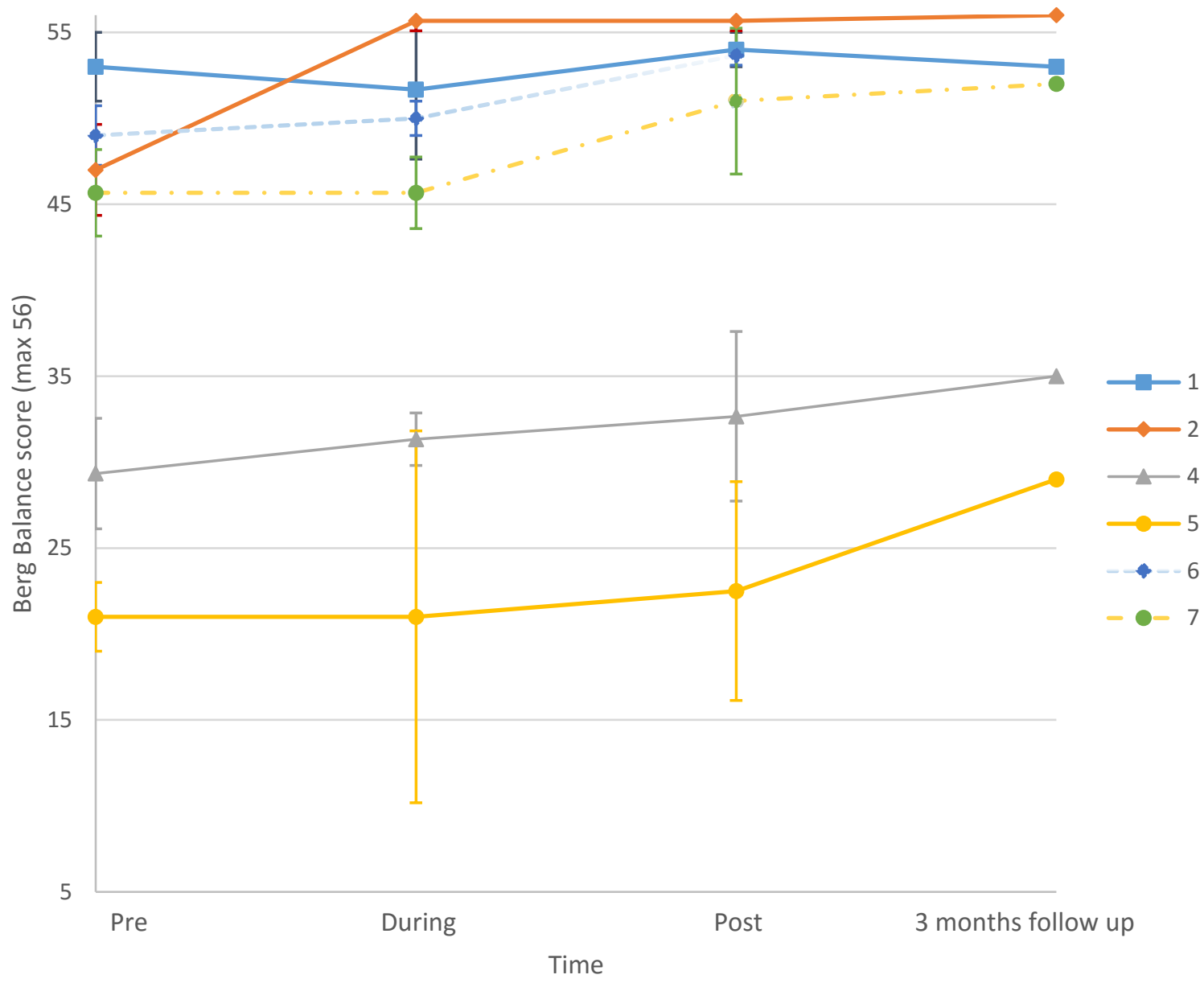

1

2 Figure 3: Berg Balance values with standard deviation bars for each participant. The pre (1), 3 during (2), and post (3) values each represent the average of three assessments. The 3 month 4 value (4) represents one assessment. Participant 6 was not able to complete the follow up 5 assessment at 3 months. 